\title{
An Improved Technique for the Histopath- ological Diagnosis and Classification of Leprosy
}

\author{
E. A. WHEELER, s.R.m.L.T., E. G. HAM I LTON F.I.M.L.T, s.R.M.L.T., and \\ D. J. HAR MAN, м.в., сн.в. Leprosy Research Unit, 57 a Wimpole Street, London, $W$. I
}

The importance of biopsies in connection with the diagnosis, prognosis and treatment of leprosy is receiving greater attention among leprologists, and it is essential therefore that the fixation, processing and staining of tissue sections should be of a high standard if the finer points in relation to diagnosis and classification of leprosy are to be appreciated.

\section{FIXATION}

It has been accepted by many that io per cent neutral formol-saline is a poor fixative for most tissues, including human skin, because of its tendency to cause considerable shrinkage of the cellular structures, and this has proved to be a hazard in assessing the histopathological picture of leprosy correctly.

In many respects Zenker's solution is the most satisfactory fixative, but the need for running water or frequent changes of water when using this method renders it almost impracticable for those working under 'bush' conditions.

We have now found that the Zenker-formol variant recommended by Dr. D. S. Ridley, Consultant Pathologist at the Hospital for Tropical Diseases, London, meets the major conditions for good fixation of skin and nerve tissue to be examined, and the details of this method as used by us are as follows:

Formula

Solution $A$ Formaldehyde 40 per cent io $\mathrm{ml}$. Mercuric chloride $2 \mathrm{gm}$. Distilled water

Solution B Glacial acetic acid.

The solution $A$ is stable and a stock bottle of this must be kept available. The glacial acetic acid $B$ is added to the solution $A$ immediately before use, that is, at the time of biopsy. For the average biopsy $20 \mathrm{ml}$. of the fixative solution is adequate and is obtained by adding $\mathrm{I} \mathrm{ml}$. of solution $B$ to $\mathrm{I} 9 \mathrm{ml}$. of solution $A$. The tissue is placed in this fixative for a minimum of 15 hours and a maximum of 24 hours. The fluid is then simply poured off and replaced by 70 per cent alcohol. After 24 hours the tissue is ready for processing, or may be despatched in the 70 per cent alcohol for processing elsewhere.

It is essential that the greatest care should be taken to prevent damage to the biopsy tissue. An excision with a sharp knife is preferable to a skin punch, and the tissue must not be crushed or pinched with forceps.

\section{P R OCESSIN G}

The biopsy tissues are processed in the usual way by dehydration through alcohols, cleared in cedar oil, and impregnated and blocked with paraffin wax. Ribbon sections are cut at 5 microns, mounted and dried in an incubator at $37^{\circ}$ Centigrade overnight.

ST AIN IN G

If the finer points of classification of leprosy are to be appreciated it is important that individual attention be given to the staining process, and we have found that a modified Masson's trichrome technique shows the details of the histopathology well and allows the physician to assess the stage of the leprosy process and to relate it to his clinical findings.

One of the drawbacks, however, has always been that the actual organism responsible for the disease, the Mycobacterium leprae, is not visible in the specimen stained by this Masson's stain, and that further sections have to be cut and stained by the Fite-Faraco modification of the Ziehl-Neelsen technique in order to see it. The changing over from one microscope slide to another is also time-consuming and in many instances inconvenient, so with this in mind it became desirable to adopt a technique for combining the above stains into one preparation, so 
that the histopathologist would be able to see the whole picture of the presence of the bacilli in the tissues and their relationship to the resultant cellular response.

Not only does this increase accuracy in the diagnosis and assessment of the case, but it makes the histopathology of leprosy much more interesting and instructive, and the preparations are excellent for study and teaching purposes. Examination of only one slide is much more convenient, and there is economy in time and materials in the preparation of specimens, and in storage space. The histopathological picture obtained is also good for colour photography and we have found no difficulty in seeing bacilli or bacillary remnants even when only one or two are present in the section. In really doubtful cases a second section can always be stained by the Fite-Faraco technique to confirm the findings.

The routine use of this staining method for biopsies of granulomatous lesions of doubtful etiology would assist in the earlier dignosis of missed leprosy cases. This method is also equally suitable for the identification of Mycobacterium tuberculosis and its resultant pathology.

As this staining method is a combination of a trichrome stain and the Fite-Faraco stain, we have temporarily dubbed it the 'Triff' method, from 'tri' to indicate trichrome and ' $\mathrm{f}$ ' to indicate Fite-Faraco. The details of the method are as follows:

\section{Triff Stain}

(Mixture of Trichrome and Fite-Faraco stains)

I. Warm slide gently to melt wax.

2. Place in pure turpentine oil for one hour.

3. Blot gently with filter paper, wash in tap water, and place in common solution of iodine ( 0.5 per cent iodine crystals in 70 per cent alcohol) for five minutes to remove mercuric salts.

4. Rinse thoroughly with tap water.

5. Immerse in I per cent aqueous solution of sodium thiosulphate until bleached - io to 20 seconds.

6. Wash in running water for five minutes.

7. Stain with cold strong filtered carbol fuchsin (Ziehl-Neelsen) for 30 minutes.

8. Wash off surplus stain with tap water for five minutes.

9. De-colourize with two washes of Fite de- colourizer (0.5 per cent hydrochloric acid in 70 per cent alcohol) rinsing with tap water in between each wash - approximately one minute each wash.

Io. Wash thoroughly with tap water for I 5 minutes.

I I. Stain with Harris' haematoxylin for two minutes.

I 2. Wash in tap water until blue.

I3. Rinse rapidly with Fite de-colourizer.

I4. Wash for at least io minutes with tap water - longer if possible.

I5. Stain with I per cent aqueous yellow eosin for 30 seconds.

I6. Wash thoroughly with tap water for io seconds followed by 70 per cent alcohol for I o seconds.

I 7. Rinse over rapidly with absolute alcohol.

I8. Stain with 2 per cent alcoholic solution of saffron* for 5 to I 5 minutes. Control with the microscope to ensure differential staining of nerves and connective tissue - a yellow colour - rinsing in alcohol to remove excess saffron and replacing in the stain if not stained sufficiently.

I 9. Rirıse rapidly with absolute alcohol.

20. Clear with xylene.

2I. Mount under a coverslip in neutral Canada balsam or DePeX.

The staining scheme is:

$\begin{array}{ll}\text { Cell nuclei } & \text { blue } \\ \text { Cytoplasm } & \text { pink } \\ \text { Muscle } & \text { pink } \\ \text { Nerve tissue } & \text { yellow } \\ \text { Connective tissue } & \text { yellow } \\ \text { Acid-fast organisms } & \text { red }\end{array}$

$\mathcal{N} . B$. The use of a known positive control section is recommended with each batch of sections stained.

It is hoped that the publication of this method will give to histopathologists a new approach to the study of the pathology of leprosy, and that it will enable them to take a greater interest in the details of the cellular changes in leprosy in relation to the presence of the Mycobacterium leprae in the tissues. Careful use of this technique will, we hope, suggest avenues of research which it would be difficult to discern by the ordinary staining methods.

$*_{2}$ per cent alcoholic solution of saffron is supplied by G. T. Gurr Ltd., I 36-1 44 New Kings Road, London, s.w.6. 
S U M M A R Y

A technique for staining paraffin sections with a combination stain of a modified Masson's trichrome and the Fite-Faraco is described in detail, and the various advantages of using this method in the diagnosis and classification of leprosy are discussed.

\section{A GKNOWLEDGEMENTS}

We would express our appreciation to Dr. Ridley for the development of a satisfactory fixative for leprosy tissues. We would also like to express our thanks to Dr. R. G. Cochrane, Director of Leprosy Research Unit, London, for his encouragement and help in the preparation of this paper. 